\title{
The mammalian heart - towards an understanding of the intrinsic antagonistic function of the ventricles
}

\author{
Niederer Peter ${ }^{1 *}$ and Lunkenheimer PP $^{2}$ \\ ${ }^{1}$ Institute of Biomedical Engineering, ETH and University of Zurich, Switzerland \\ ${ }^{2}$ Department of Experimental Thoraco-vascular Surgery, University of Muenster, Germany
}

\begin{abstract}
The mammalian myocardium making up the ventricles exhibits, on a microscopic scale, a lamellar structure. Lamellae consist of end-to-end connected myocyes in the form of long strains that are densly crosslinked by bridging myocytic offsprings and strongly bound by the endomysium. In contrast, between lamellae only weak myocytic crosslinking and sparse connective tissue is found. The latter property enables reorientation of lamellar segments during systole. Due to the overall crosslinking, however, the myocardium represents a continuum, albeit with significant local variation. While most lamellar segments are oriented and contract during systole in a mostly surface-paralell direction, enabling the ejection of blood, there is a notable presence of transversely oriented lamellar segments. Such segments develop a partly antagonistic function (with respect to the one of the surface parallel segments), in that they counteract systolic wall thickening, the major mechanism for the ejection of blood. This so far underestimated intrinsic antagonic organisation is substantiated by anatomic analyses; furthermore, significant consequences with respect to cardiodynamics and diseases of the heart are found.
\end{abstract}

\section{Introduction}

Antagonism is a basic feature in biology manifesting itself ubiquitously. e.g., coagulation of blood is antagonized by hemolysis, efferent and afferent nerves act antagonistically, etc. In particular, muscles, acting in essence unidirectionally, are usually associated with an antagonist.

In contrast, the four-chamber structure heart muscle seems to be void of an antagonistic counterpart at first sight. Its apparent function consists of ejecting blood, an antagonistic function seems to be unnecessary. Yet, an in-depth analysis of anatomy and function of the mammalian ventricles reveals that a particular form of antagonism is intrinsically present and exhibits important properties in view of physiologically well-ordered cardiodynamics.

Due to its physiological (also clinical) dominance, the left ventricle is in the foreground of our considerations. The anatomy of the human ventricles, along with their mechanical function (cardiodynamics) has been subject of research through centuries. Yet, there are still unresolved issues in this field. Well established is the fact that the human myocardium exhibits on a microscopic scale a lamellar structure [1]. Lamellae are thereby composed of long strains of multi-branching cardiomyocytes and are highly diverse with respect to dimensions. While lateral dimensions are generally in the sub-millimeter range, the length of individual lamellae is virtually unlimited; lamellae can be followed around the entire ventricle along numerous paths generated by inter-lamellar branching. Lamellae are intrinsically strongly bound by the dense endomysium and cardiomyocytes may have up to 7 offsprings connecting adjacent strains. Frequent inter-lamellar branching connections also exist, but bondage is quite loose [2-4]. The inter-lamellar perimysium and cross-bridges thereby allow for appreciable mutual displacement during the heart cycle (cleavadge planes). Further constituents of the myocardium consist of the electrical conduction system, the intracellular matrix and the vasculature.
Likewise, well established is the general global helical architecture of the left ventricle: A helical arrangement of the lamellar units from the base to the apex is dominating.

There exists some controversy however with respect to the intermediate structure of the myocardium, i.e. between the global and macroscopic and microscopic scale. This bears enormous significance for cardiodynamics. Two main conceptual architectures have been proposed in the past. Most often put forward is the notion that all cardiomyocytes have a spatial orientation that is in essence surfaceparallel $[5,6]$. The systolic action of the heart seems to support this view as a natural premise since the cardiomyocytes act essentially as uniaxial contraction elements. Within the framework of a further concept, also apparently suggested by the systolic constriction property of the ventricles, a band-like structure of the myocardium has been postulated [7]. Yet, both concepts can not be supported by either anatomical facts or cardiodynamics' considerations. The myocardium is best understood as a netted continuum with substantial local variability; in fact, due to lamellar crosslinking by myocytic offsprings, every location in the ventricular wall can be reached without leaving myocytic strains.

\section{Ventricular anatomy}

Gentle pneumatic distention of the excised ventricles allows to visualize the cleavadge system by CT [8] and outline the myocardial architecture (Figure 1).

${ }^{*}$ Correspondence to: Niederer Peter, Institute of Biomedical Engineering, ETH and University of Zurich, Switzerland, E-mail: niederer@biomed.ee.ethz.ch

Key words: heart, myocardium, intrinsic antagonism, cardiodynamics

Received: October 24, 2018; Accepted: November 20, 2018; Published: November 23, 2018 

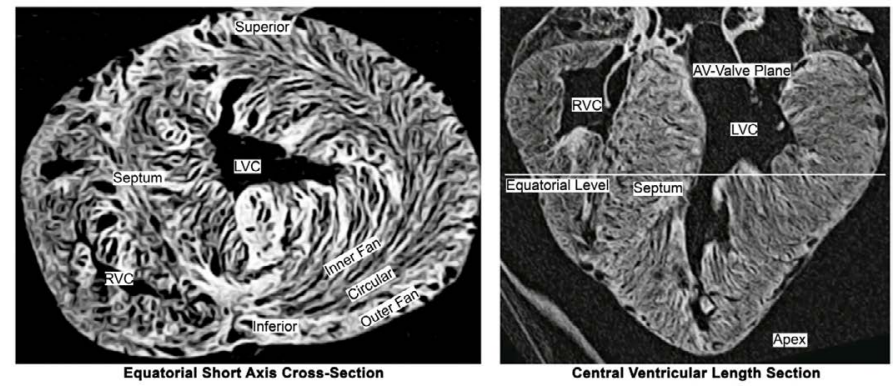

Figure 1. Computer-Tomographic cross-section (left) and length-section (right) through a pneumatically slightly distended porcine heart, which expose its layered lamellar structure. It is found to vary widely between regions

Quantification of the spatial orientation of lamellar segments in the $\mathrm{mm}$ range shows that deviations of more than $30^{\circ}$ degrees in diastole and 40 degrees at end-systole from a surface-parallel orientation are observed in up to $40 \%$ of all discernable segments [9-12] (the wellknown helical $\pm 60^{\circ}$ distribution of the myocytes' deviation from a plane perpendicular to the long axis is also confirmed). A strong stochasiticity is furthermore associated with the local orientation distribution as well as with the ventricular inner and outer surface. At first sight, some similarity with a bird's nest becomes apparent (Figure 2).

\section{Cardiodynamics}

\section{Antagonistic action within the ventriculae wall}

There are two major cardiodynamic causes that underline the significance of the findings outlined above. First, lamellar segments, more precisely cardiomyocytes, having a circular, surface-parallel orientation provide in essence the thrust for the systolic ejection of blood. As a result of circular constriction, mural thickening is induced that in fact is one major mechanism causing ejection. Contractile elements, however, that exhibit a strong deviation from a surfaceparallel direction, produce a constrictive force component in radial direction, thereby counteracting mural thickening [13]. The effect consists of a significant antagonistic (with respect to the circular constriction) action. The two components generate different types of force tracings when measured by miniaturized intramural forceprobes (Figure 3). In agreement with Starling's law, lamellar segments that are oriented in a surface parallel alignment, unload during systolic shortening. In contast, the forces produced by lamellar segments in a transmural arrangement increase as such segments elongate in the course with mural thickening.

There is evidence, furthermore, that the antagonism represents an important determinant for keeping an overall internal balance of forces and deformations (inertial effects can largely be disregarded), avoid excessive deformations and monitor cardiac performance into early diastole (antagonistic forces were found to extend into early diastole thereby supporting rapid early diastolic filling). Animal experiments involving inotropic drugs and clinical observations (below) in connection with fibrosis confirm the antagonistic concept [14].

Second, an apparently "optimal", regular arrangement of cardiomyocytes has been analysed in mathematical heart models; such arrangements, however, are found to be highly sensitive and unphysiologic: even a slight deviation from the "optimal" architecture results in a dramatic decrease of cardiac performance. Stochasticity, in turn, enables a stable cardial performance over a wide variety of anatomical aberrations $[15,16]$.

\section{Asymmetry of the action of inotrops}

It is a specific feature of the oblique transmural netting component to react more sensitive to inotropic interventions than the tangential netting component. The answer of the oblique structure to positive inotropics is at least double that of the tangential alignment. The answer to negative inotropics is 5 times more pronounced (Figure 4). In any case this asymmetry in answer only apply to low dosage of medication. At high doses the answer to positive as well to negative inotrops more or less is comparable in both netting components $[13,17,18]$.

\section{Clinical consequences}

The derailment of the equilibrium provided by the antagonistic organisation of myocardial function might explain various cardiological diseases. Thereby, the clinical understanding of ventricular afterload has to be extended. Besides the extrinsic hemodynamic afterload, the heart contracts against an intrinsic myostructural, stromatogenic and hydraulic resistance. Within the framework of this complex afterload environment that the heart has to cope with, it is prone to derail each in a charcteristic way, causing each a particular pattern of clinical pathology and requiring each its particular therapy [14].

\section{Hemodynamic Afterload}

The hemodynamic afterload is given by the systolic ventricular pressure, typically increases as a consequence of arterial hypertension,

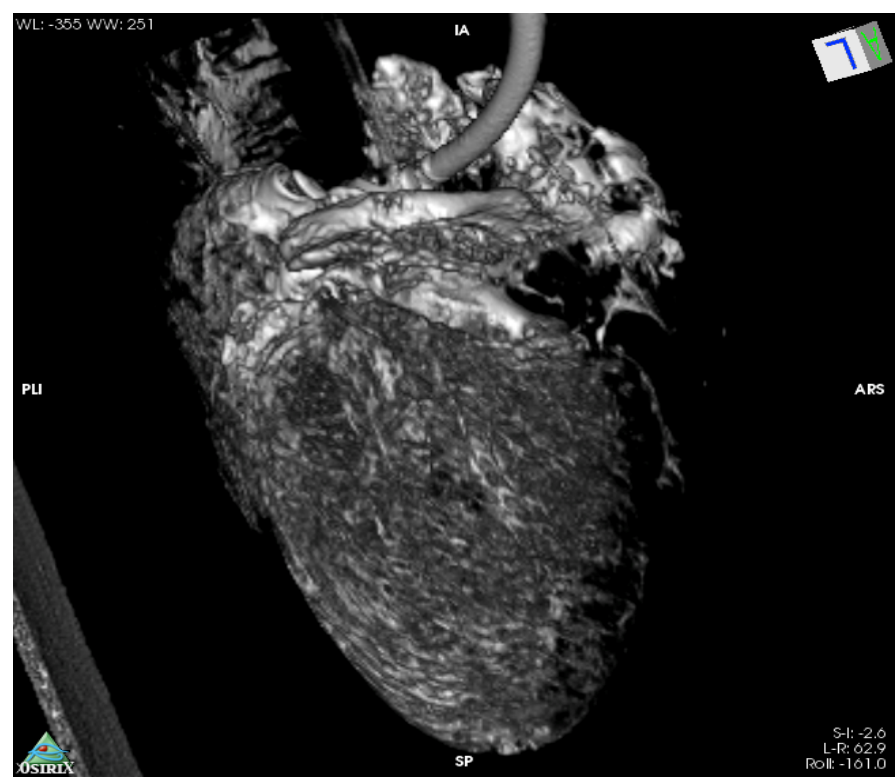

Figure 2a. Semitransparent view of the lamellar structure of the myocardium

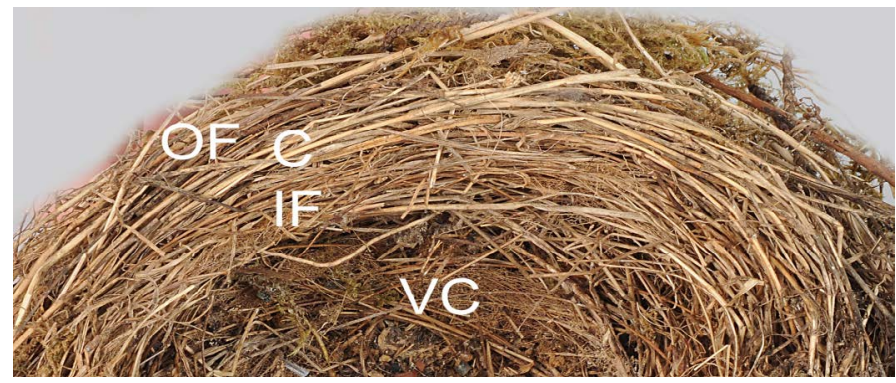

Figure 2b. Section from the wall of a bird's nest that resembles in the different netting of the blades of grass in the outer fan (OF), circular layer (C) and inner fan (IF) that of the heart's wall. The centre of the nest represents the cavity of the ventricle (VC) 


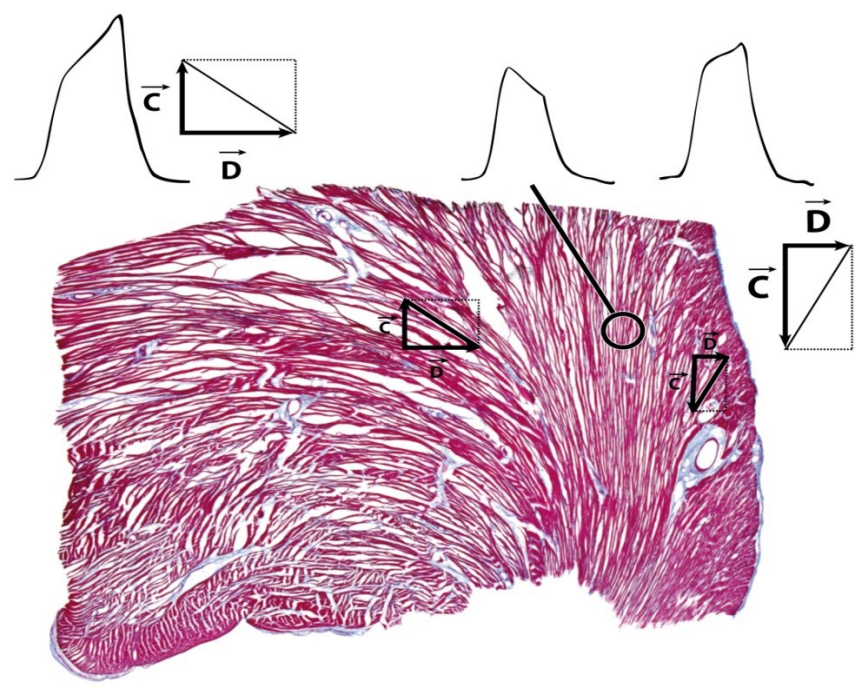

Figure 3. Two types of force are measured within the spatially netted myocardium: The upper left and the upper right tracings show "auxotonic" forces, when the force probe is coupled to oblique transmural netting components. Systolic rise in force increases with the degree of deviation from the tangential alignment. The middle tracing shows an "unloading" signal, measured in the tangential netting component [13]

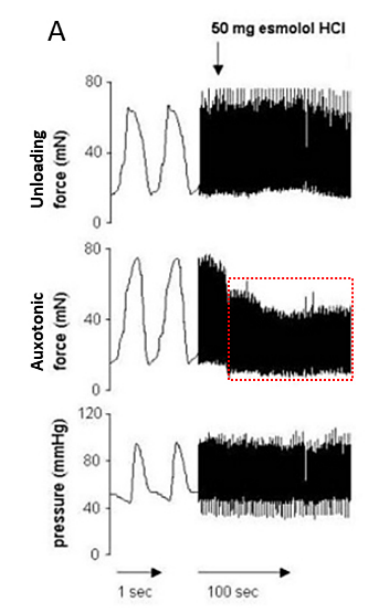

B

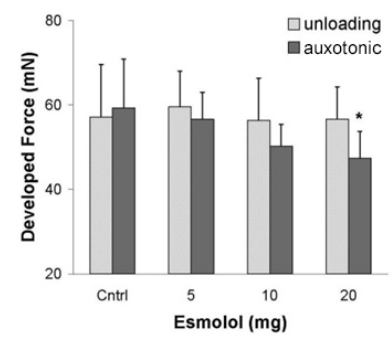

Figure 4. The asymmetric effect of the $\beta$-Blocker esmolol following increasing doses on the force tracing of tangential structures (unloading signal) and on the forces generated by oblique transmural netting (auxotonic signal)

aortic stenosis or contractile or fibrotic constriction of the ventricular outflow tract. Obviously, those disturbances can well be diagnosed and treated under control of intracavitary pressure.

\section{Myostructural Afterload}

The myostructural afterload which results from myocardial transmural netting, derails in all kinds of myocardial hypertrophy. The particular feature of hypertrophy is that the intrinsic antagonism sustains the process of hypertrophy such the disease is self-amplifying. This vicious circle can be interrupted by tempering separately the contractile forces in the oblique transmural deviation of myocardial netting, which can be achieved by use of low dose negative inotropics. The clinician's problem is that there are no means to evaluate the degree of derailment unless by measuring wall stiffness, which recently promises to become measurable in terms of "Cardiac Shear Wave Velocity Detection" [19-21].
Another manifestation of derailment of myostructural derailment most probably is Takotsubo-syndrome [22-24], which goes along with an active systolic dilation of the ventricular apex. Concommittant increase in proximal wall tension, which again escapes clinical evaluation, restricts perfusion of the intramural microvasculature and ends up in most painful myocardial ischemia. Running investigations are focused on the question, whether the dominance of dilating forces is caused by an atrophy of tangential netting components in physically undertrained elderly patients, while the oblique transmural netting component persists because it is kept fighting against systolic wall thickening. An alternative option is an excessive increase in sensitivity of transmural netting components for stress-induced positive inotropic stimulation. In any case the therapeutic way to go is low dose $\beta$-Blockade [25].

\section{Stromatogeni afterload}

The stromatogenic afterload derives from the effect of connective tissue netting which in the normal heart most probably has no measurable effect. It increases however dramatically in case of transmural or diffuse myocardial fibrosis (Figure 5) which hinders systolic wall thickening. At the time being there is no effective therapy described.

\section{Hydraulic afterload}

The hydraulic afterload is caused by intramural fluid pressure, which increases in case of myocardial edema in myocarditis and in myocardial storage diseases. Intramural pressure acts on the smaller endocardial surface plane as well as on the larger epicardial surface plane which means that in any case intramural pressure has a prevailing dilatory effect (Figure 6). With any kind of accumulation of intramural fluids, wall thickness increases, which event expands the surface difference between epicardium and endocardium. Furthermore, intramural pressure increases. All effects contribute to an increase in hydraulic afterload hence dilating forces increase. Therapy must focus on reduction in wall thickness and intramural pressure by eliminating fluid and stored semifluid masses.

\section{Conclusions}

The ventricular architecture is characterized by a lamellar structure that is ubiquitously crosslinked. While during systole constrictive

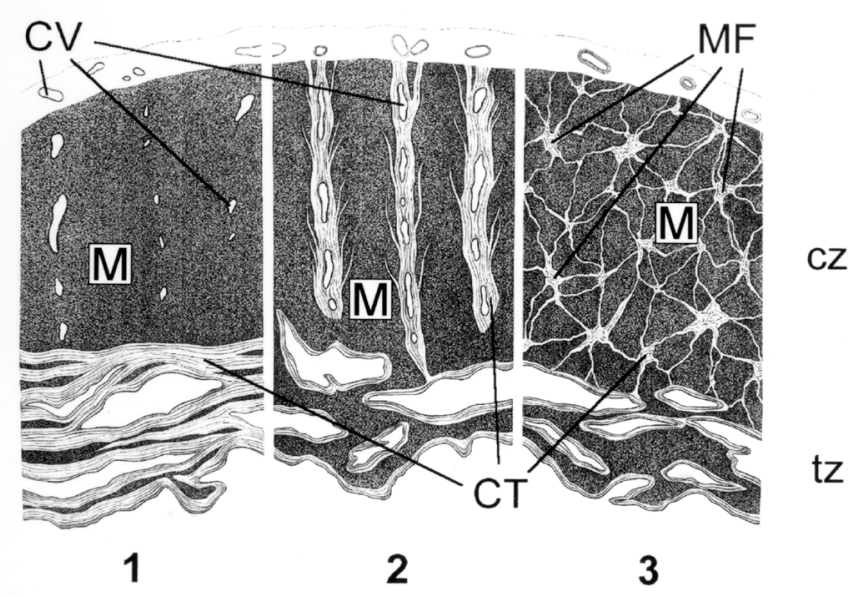

Figure 5. 1: tangential scaring; 2: regional transmural scaring; 3: three-dimensional microfocal scaring. Related diseases are Global Myocardial Fibrosis, regional Cicatrizing CV: Coronary Vessels; M: Myocardium; MF: Micro-Focal Scars; CT: Connective Tissue; cz: Compact Zone; tz: Trabeculated Zone 


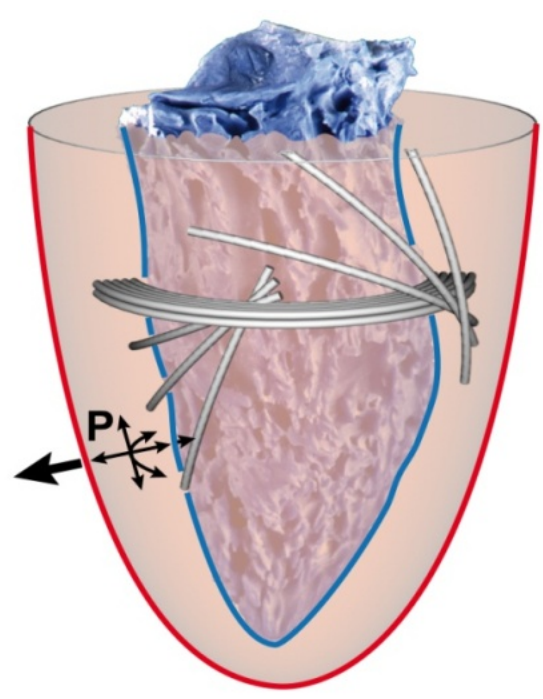

Figure 6. Epicardial Surface-Plane (red) $>$ Endocardial Surface-Plane (blue). Related Diseases: Morbus Fabry, Amyloidose, Myocardial Edema, Myocardial Hypertrophy (wall thickening)

(essentially surface parallel) forces causing the ejection of blood dominate, there exists a notable antagonistic, dilating activity (opposing mural thickening) caused by lamellar segments that are oriented in a transmural direction. This antagonistically organized setting, along with some stochasticity with respect to lamellar segment orientation, is well adapted to keep local balance in that excessive deformation patterns are prevented; furthermore, a ventricle working properly over a wide range of physiological conditions is supported.

Derailment of such antagonistic equilibration is prone to cause diseases that manifest themselves in various forms. In particular, in myocardial hypertrophy, disturbances in fibre-netting like obstructive hypertrophic cardiomyopathy, fibrosis, edema, storage diseases and, as described only recently, in Takotsubo-syndrome, which causes critical increase in antagonistic wall stress. This new pattern of pathophysiology requires new diagnostic means by which to measure wall stiffness as an indicator of wall tension.

\section{References}

1. LeGriece IJ, Smail BH, Chai LZ, Edgar SG, JGavin JB, et al. (1995) Laminar structure of the heart: ventricular myocyte arrangement and connective tissue architecture in the dog. Am J Physiol 269: H571-H582. [Crossref]

2. Niederer P, Lunkenheimer PP, Cryer CW (2004) On the significance of fiber branching in the human myocardium. Biomech Model Mechaniobiol 3: 1-5. [Crossref]

3. Harrington KB, Rodriguez F, Cheng A, Langer F, Ashikaga H, et al. (2005) Direct measurement of transmural laminar architecture in the anterolateral wall of the ovine left ventricle: new implications for wall thickening mechanics. Am J Physiol Heart Circ Physiol 288: H1324-H1330. [Crossref]

4. Robinson ThF, Cohen-Gould L, Factor SM (1983) The skeletal framework of mammalian heart muscle: arrangement of inter- and pericellular connective tissue structures. Lab Invest 49: 482-498. [Crossref]

5. Frank O (1901) Isometrie und Isotonie des Herzmuskels. Z Biol 41: 14-34.

6. Mall FP (1911) On the muscular architecture of the ventricles of the human heart. $\mathrm{Am}$ J Anat 11: 211-266.

7. Torrent-Guasp F (1980) La estructuracion macroscopica del miocardio ventricular. Rev Esp Cardiol 33: 265-287.

8. Lunkenheimer PP, Müller RP, Konermann Chr, Lunkenheimer A, Köhler F (1984) Architecture of the myocardium in computer-tomography. Invest Radiol 19: 271-278. [Crossref]
9. Lunkenheimer PP, Redmann K, Westermann PH, Rothaus K, Cryer CW, et al. (2006) The myocardium and its fibrous matrix working in concert as a spatially netted mesh: a critical review of the purported tertiary structure of the ventricular mass. Eur J CardioThorac Surg 295: 41-49. [Crossref]

10. Lunkenheimer PP, Redmann K, Kling N, Jiang X, Rothaus K, et al. (2006) Threedimensional architecture of the left ventricular myocardium. Anat Rec A Discov Mol Cell Evol Biol 288: 565-578. [Crossref]

11. Schmid P, Lunkenheimer PP, Redmann K, Rothaus K, Jiang X, et al. (2007) Statistica analysis of the angle of intrusion of porcine ventricular myocytes from epicardium to endocardium using diffusion tensor magnetic resonance imaging. Anat Rec (Hoboken) 290: 1413-1423. [Crossref]

12. Smerup M, Agger P, Nielsen EA, Ringgaard St, Pedersen M, et al. (2013) Regiona and Epi-to Endocardial Differences in Transmural Angles of Left Ventricular Cardiomyocytes Measured in Ex Vivo Pig Hearts: Functional Implications. Anat Rec (Hoboken) 296: 1724-1734. [Crossref]

13. Lunkenheimer PP, Redmann K, Florek J, Fassnacht U, Cryer CW, et al. (2004) The forces generated within the musculature of the left ventricular wall. Heart 90: 2002007. [Crossref]

14. Lunkenheimer PP, Niederer P, Stephenson RS, Redmann K, Batista RV, et al. (2018) What is clinical significance of ventricular mural antagonism? Eur $J$ Cardiothor Surgery 53: 714-723. [Crossref]

15. Dorri F, Niederer P, Redmann K, Lunkenheimer PP, Cryer CW, et al. (2007) An analysis of the spatial arrangement of the myocardial aggregates making up the wall of the left ventricle. Eur J Cardio Thorac Surg 31: 430-437. [Crossref]

16. Dorri F, Niederer P, Lunkenheimer PP (2006) A finite element model of the left ventricular systole. Comput Methods Biomech Biomed Engin 9: 319-341. [Crossref]

17. Lunkenheimer PP, Redmann K, Isringhaus H, Flameng W, Rettig G, et al. (1992) Cardiodynamic effects of dopamine and dobutamine. Thorac Cardiovasc Surg 40: 135139. [Crossref]

18. Lunkenheimer PP, Redmann K, Cryer CW, Batista RIV, Stanton JJ, et al. (2007) Betablockade at low doses restoring the physiological balance in myocytic antagonism. Eur J Cardio Thorac Surg 32: 225-230. [Crossref]

19. Bhatia K, Tong CSL, Cho CCM, Yuen EHY, Lee J, et al. (2012) Reliability of Shear Wave Ultrasound Elastography for neck lesions identified in routine clinical practice. Ultraschall Med 33: 463-468. [Crossref]

20. Urban MW, Pislaru C, Nenadic IZ, Kinnik RR, Greenleaf JF (2013) Measurement of viscoelastic properties of in vivo swine myocardium using lamb wave dispersion ultrasound vibrometry (LDUV). IEEE Trans Med Imaging 32: 247-261. [Crossref]

21. Vos HJ, van Dalen BM, Heinonen I, Bosch JG, Sorop O, et al. (2017) Cardiac Shear Wave Velocity Detection in the Porcine Heart. Ultrasound Med Biol 43: 753-764. [Crossref]

22. Sato H, Tateishi H, Uchida T, et al. (1990) Takotsubo type cardiomyopathy due to multivessel spasm. In: Kodama K, Haze K, Hon M, editors. Clinical aspect of myocardial injury: from ischemia to heart failure. Kagaku Hyoronsha; Tokyo: 56-64.

23. Kurisu S, Sato H, Kawagoe T, Ishihara M, Shimatani Y, et al. (2002) Tako-tsubo-like left ventricular dysfunction with ST-segment elevation: a novel cardiac syndrome mimicking acute myocardial infarction. Am Heart J 143: 448- 455. [Crossref]

24. Bybee KA, Kara T, Prasad A, Lerman A, Barsness GW, et al. (2004) Systematic review: transient left ventricular apical ballooning: a syndrome that mimics ST-segment elevation myocardial infarction. Ann Intern Med 141: 858-865. [Crossref]

25. Angue M, Soubirou L, Vandroux D, Cordier C, Martinet O, et al. (2014) Beneficial effects of intravenous beta-blockers in Tako-Tsubo syndrome with dynamic left ventricular outflow tract obstruction and severe haemodynamic impairment. Int $J$ Cardiol 177: 56-57. [Crossref]

Copyright: (C2018 Peter N. This is an open-access article distributed under the terms of the Creative Commons Attribution License, which permits unrestricted use, distribution, and reproduction in any medium, provided the original author and source are credited. 